\title{
A CONJECTURE ON A CONTINUOUS OPTIMIZATION MODEL FOR THE GOLOMB RULER PROBLEM
}

\author{
Phil Duxbury ${ }^{1}$, Carlile Lavor ${ }^{2}$ and Luiz Leduino de Salles-Neto ${ }^{3, *}$

\begin{abstract}
A Golomb Ruler (GR) is a set of integer marks along an imaginary ruler such that all the distances of the marks are different. Computing a GR of minimum length is associated to many applications (from astronomy to information theory). Although not yet demonstrated to be NP-hard, the problem is computationally very challenging. This brief note proposes a new continuous optimization model for the problem and, based on a given theoretical result and some computational experiments, we conjecture that an optimal solution of this model is also a solution to an associated GR of minimum length.
\end{abstract}

Mathematics Subject Classification. 90C26, 90C27.

Received March 31, 2021. Accepted July 11, 2021.

\section{INTRODUCTION}

Given a positive integer $n$, the Golomb Ruler Problem (GRP) asks for locating $n$ integer marks along an imaginary ruler such that all distances between pairs of marks are distinct and the length of the ruler is minimum [3,6]. The GRP has many applications, including astronomy [4], communications [1], and information theory [18].

Historically, the GRP first appeared related to Sidon sets [21], i.e. sets of integers so that all pairwise sums of elements of the set are distinct. Lower bounds for the GRP and connections to number theory are discussed, respectively, in $[7,14,22,23]$.

We say that we have a Golomb Ruler (GR), given $n$ marks, when the associated distances are all different, and an Optimal Golomb Ruler (OGR), when a GR has the smallest length.

For example, the OGR for $n=4$ is $\{0,1,4,6\}$, and for $n=5$, there are two solutions: $\{0,1,4,9,11\}$ and $\{0,2,7,8,11\}$.

Although not yet demonstrated to be NP-hard, solving the GRP exactly proved to be very difficult $[5,19]$. Solutions for all $n \leq 27$ are available on Wikipedia ("Golomb Ruler", acessed on February 15th, 2021).

In [13], a linear programming model is proposed for the GRP and, more recently, authors in [8] compare different optimization approaches to certify optimality to the GRP.

Keywords. Nonlinear programming, Golomb Ruler Problem, continuous models.

1 Michigan State University, Natural Science Building, 288 Farm Lane, East Lansing, MI, USA.

2 University of Campinas (IMECC - Unicamp), Cidade Universitaria Zeferino Vaz, Campinas, Brazil.

3 Federal University of Sao Paulo, Science and Technology Institute, Sao Jose dos Campos, Brazil.

*Corresponding author: luiz.leduino@unifesp.br

(c) The authors. Published by EDP Sciences, ROADEF, SMAI 2021 
We present a new model to the GRP based on a continuous optimization formulation (Sect. 2.1). In Section 2.2, we give a theoretical result and conjecture that a solution of this model is also a solution to the associated GRP. Computational experiments are given in Section 3. Section 4 concludes the paper with new research directions.

\section{Models, A theoretical Result And a COnjecture for the GRP}

Formally, a GR consists of a set of integers $R=\left\{r_{1}, \ldots, r_{n}\right\}$, with $r_{1}<r_{2}<\ldots<r_{n}$, such that for each positive integer $d$, there is at most one pair $\left\{r_{i}, r_{j}\right\} \subset R, i<j$, that satisfies

$$
d=r_{j}-r_{i}
$$

The set $R$ represents positions of $n$ marks on the ruler with length $L=r_{n}-r_{1}$. Without loss of generality, we fix $r_{1}=0$.

\subsection{A new model for the GRP}

In order to present the first model to the GRP (first described in [8]), we need an upper bound $L_{u}$ for the length of the ruler, given also as input of the problem, in addition to $n$ marks on the ruler:

$$
\begin{array}{rlr}
\min _{t \geq 0, x_{i} \in\{0,1\}} & t \\
\text { s.t. } & & \\
& i x_{i} \leq t, & \\
& \sum_{i=1}^{L_{u}} x_{i}=n-1, \ldots, L_{u}, \\
& x_{j}+\sum_{i=1}^{L_{u}-j}\left(x_{i} x_{i+j}\right) \leq 1, & \\
& x_{i} \in\{0,1\}, & \\
& &
\end{array}
$$

Since we are minimizing $t$, inequalities

$$
i x_{i} \leq t
$$

for $i=1, \ldots, L_{u}$, "force" the length of the ruler to be minimum. At the same time, equation

$$
\sum_{i=1}^{L_{u}} x_{i}=n-1
$$

requires that $n-1$ of the variables $x_{i}$ are equal to 1 , and inequalities

$$
x_{j}+\sum_{i=1}^{L_{u}-j}\left(x_{i} x_{i+j}\right) \leq 1,
$$

for $j=1, \ldots, L_{u}-1$, guarantee that all distances between pairs of marks are distinct.

A solution $\left(x_{1}, \ldots, x_{L_{u}}\right)$ of model $(2.1)$ provides a GRP solution given by $\left(i x_{i}\right)$, for $i=1, \ldots, L_{u}$, whose minimum length of the associated ruler is the optimal value found for $t$. 
In the literature, there are many heuristics for calculating upper bounds for the GRP (e.g. see [8]). We propose a simple greedy heuristic whose pseudocode is given below ${ }^{1}$ :

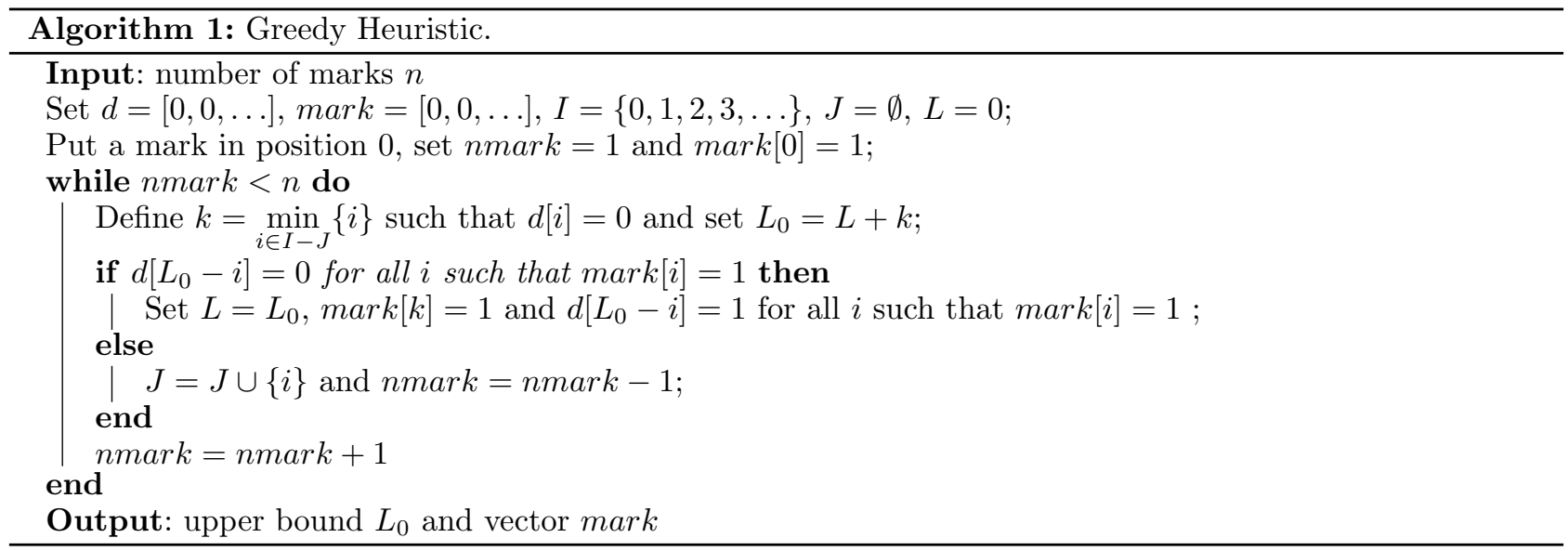

To solve larger instances of the GRP, compared to model (2.1), we propose below a continuous version of this model, with a simple but fundamental modification on the first constraint:

$$
\begin{aligned}
& \min _{t \geq 0, x_{i} \in[0,1]} t \\
& \text { s.t. } \\
& i x_{i} \leq t x_{i}, \quad i=1, \ldots, L_{u}, \\
& \sum_{k=1}^{L_{u}} x_{i}=n-1 \text {, } \\
& x_{j}+\sum_{i=1}^{L_{u}-j}\left(x_{i} x_{i+j}\right) \leq 1, \quad j=1, \ldots, L_{u}-1, \\
& 0 \leq x_{i} \leq 1, \quad i=1, \ldots, L_{u} .
\end{aligned}
$$

\subsection{A theorem and a conjecture for the GRP}

The next result establishes that, if the optimal solution $\left(x_{1}, \ldots, x_{L_{u}}\right)$ of model $(2.2)$ is such that $x_{i} \in\{0,1\}$, for $i=1, \ldots, L_{u}$, in fact, $\left(x_{1}, \ldots, x_{L_{u}}\right)$ is also a solution for the associated GRP.

Theorem 2.1. Given an upper bound $L_{u}$ for model (2.2) and let $L$ be the optimal value associated to an optimal solution $x=\left(x_{1}, \ldots, x_{L_{u}}\right)$. If $x_{i} \in\{0,1\}$, for $i=1, \ldots, L_{u}$, then $x=\left(x_{1}, \ldots, x_{L_{u}}\right)$ is an optimal solution for model (2.1) with optimal value equal to $L$.

Proof. If $x=\left(x_{1}, \ldots, x_{L_{u}}\right)$ is an optimal solution to problem $(2.2)$ with $x_{i} \in\{0,1\}$, for $i=1, \ldots, L_{u}$, then $x=\left(x_{1}, \ldots, x_{L_{u}}\right)$ is a feasible solution to problem (2.1). Suppose that there exists another feasible solution $y=\left(y_{1}, \ldots, y_{L_{u}}\right)$ to problem $(2.1)$ such that $t=\max _{i=1, \ldots, L_{u}}\left\{i y_{i}\right\}<L$. Since $y$ is a feasible solution to problem (2.2), $L$ cannot be the optimal value, which is a contradiction.

Computational experiments provided in the next section indicate that the opposite direction of the above result is also true, which we state as a conjecture, given as follows.

Conjecture 2.2. Given an upper bound $L_{u}$ for model (2.1), if $L$ is the optimal value associated to an optimal solution $x=\left(x_{1}, \ldots, x_{L_{u}}\right)$, then $x=\left(x_{1}, \ldots, x_{L_{u}}\right)$ is an optimal solution for model (2.2) with optimal value equal to $L$.

\footnotetext{
${ }^{1}$ The source code is available on GitHub at https://github.com/luizleduino/golombruler/blob/master/heuristic.
} 
TABLE 1. Upper bounds.

\begin{tabular}{llll}
\hline \hline$n$ & Heuristic & $n^{2}$ & $n^{2}-n$ \\
\hline 5 & 12 & 25 & 20 \\
6 & 20 & 36 & 30 \\
7 & 30 & 49 & 42 \\
8 & 44 & 64 & 56 \\
9 & 59 & 81 & 72 \\
10 & 75 & 100 & 90 \\
11 & 96 & 121 & 120 \\
12 & 118 & 144 & 132 \\
13 & 143 & 169 & 156 \\
14 & 169 & 196 & 182 \\
15 & 197 & 225 & 210 \\
16 & 230 & 256 & 240 \\
17 & 264 & 289 & 272 \\
18 & 299 & 324 & 306 \\
19 & 335 & 361 & 342 \\
20 & 373 & 400 & 380 \\
21 & 413 & 441 & 420 \\
22 & 455 & 484 & 462 \\
23 & 501 & 529 & 506 \\
24 & 549 & 576 & 552 \\
25 & 598 & 625 & 600 \\
26 & 648 & 676 & 650 \\
27 & 701 & 729 & 702 \\
28 & 758 & 784 & 756 \\
\hline & & & \\
\hline
\end{tabular}

\section{Computational Results}

For $n=5, \ldots, 28$, the values for $L_{u}$ were obtained using the greedy heuristic as described in the previous section. Table 1 gives such values, comparing to two conjectured upper bounds for the GRP [8].

For $n=5, \ldots, 11$, Table 2 shows the computational time, in seconds, required to find the associated optimal values (also given) considering models (2.1) and (2.2).

Model (2.2) was solved using the software "Knitro 12.3.0", via Neos Server Version $6.0^{2}$, and "Cplex 12.8.0" was used to solve model (2.1), since it is better than Knitro to combinatorial problems. All the instances run on a Lenovo laptop with $6 \mathrm{~GB}$ RAM, intel celeron $1.6 \mathrm{GHz}$ and 64 bit windows 10 .

The limit time was set to $120000 \mathrm{~s}$ when the solver Cplex was used. For the solver Knitro, we used 5000 random initial solutions.

From Table 2, we see that the computational time required for solving model (2.1) increases exponentially and no solution is found for $n>7$.

\section{Conclusions}

Using a very modest computer, small-size instances of the GRP were solved, with related computational time increasing sub-exponentially (differently from the exponential cost of the combinatorial model), which may suggest promising research directions based on this new approach. Indeed, it is possible to develop an ad hoc nonlinear optimization algorithm for the mathematical model proposed,in order to improve the computational

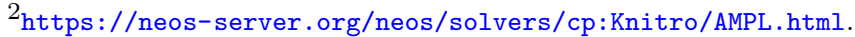


TABLE 2. Computational time in seconds and optimal values.

\begin{tabular}{lllll}
\hline \hline$n$ & $L_{u}$ & Optimal Value & Model $(2.1)-$ Cplex & Model $(2.2)-$ Knitro \\
\hline 5 & 12 & 11 & 0.125 & 1.66 \\
6 & 20 & 17 & 0.266 & 4.30 \\
7 & 30 & 25 & 395.265 & 11.37 \\
8 & 44 & 34 & - & 18.78 \\
9 & 59 & 44 & - & 299.14 \\
10 & 75 & 55 & - & 579.84 \\
11 & 96 & 72 & - & 589.30 \\
\hline
\end{tabular}

results. This possibility can be considered the main advantage of our proposal, compared to the other approaches given in literature.

Compared to two conjectured upper bounds for the GRP, the proposed greedy heuristic also presented good results as observed in Table 1.

Our approach is based on a continuous optimization model and we conjecture that an optimal solution of this model is also a solution to the related GRP.

We emphasize that GRP solutions for $n=24,25,26,27$ have been obtained by a massively parallel computational system (www.distributed.net) and the problem for $n=28$ is currently under way, whose search started in 2014 .

Motivated by the application of the GRP on wireless localization [17] and Partial Digest Problem [15], the connection with Distance Geometry $[2,9-12,16]$ should be exploited, also considering the variants of the GRP in $2 \mathrm{D}$ or higher dimensions [20].

Acknowledgements. We would like to thank the Brazilian research agencies CNPq, CAPES, and FAPESP for their financial support and the reviewer's comments.

\section{REFERENCES}

[1] W. Babcock, Intermodulation interference in radio systems. Bell Labs Tech. J. 32 (1953) 63-73.

[2] S. Billinge, P. Duxbury, D. Gonçalves, C. Lavor and A. Mucherino, Recent results on assigned and unassigned distance geometry with applications to protein molecules and nanostructures. Ann. Oper. Res. 271 (2018) 161-203.

[3] G. Bloom and S. Golomb, Applications of numbered undirected graphs. Proc. IEEE 65 (1977) 562-570.

[4] E. Blum, J. Ribes and F. Biraud, Some new possibilities of optimum synthetic linear arrays for radioastronomy. Astron. Astrophys. 41 (1975) 409-411.

[5] A. Dollas, W. Rankin and D. McCracken, A new algorithm for Golomb ruler derivation and proof of the 19 mark ruler. IEEE Trans. Inf. Theory 44 (1998) 379-382.

[6] K. Drakakis, A review of the available construction methods for Golomb rulers. Adv. Math. Commun. 3 (2009) $235-250$.

[7] P. Erdös and P. Turán, On a problem of Sidon in additive number theory and on some related problems. J. London Math. Soc. 16 (1941) 212-215.

[8] B. Kocuk and W.-J. van Hoeve, A computational comparison of optimization methods for the Golomb Ruler Problem. Lecture Notes Comput. Sci. 11494 (2019) 409-425.

[9] C. Lavor, L. Liberti, W. Lodwick and T. Mendonça da Costa, An Introduction to Distance Geometry Applied to Molecular Geometry. SpringerBriefs, Springer, New York (2017).

[10] L. Liberti and C. Lavor, Six mathematical gems from the history of distance geometry. Int. Trans. Oper. Res. 23 (2016) $897-920$.

[11] L. Liberti and C. Lavor, Euclidean Distance Geometry: An Introduction. Springer, New York (2017).

[12] L. Liberti, C. Lavor, N. Maculan and A. Mucherino, Euclidean distance geometry and applications. SIAM Rev. 56 (2014) 3-69.

[13] R. Lorentzen and R. Nilsen, Application of linear programming to the optimal difference triangle set problem. IEEE Trans. Inf. Theory 37 (1991) 1486-1488.

[14] C. Meyer and B. Jaumard, Equivalence of some LP-based lower bounds for the Golomb Ruler Problem. Discrete Appl. Math. 154 (2006) 120-144. 
[15] C. Meyer and P. Papakonstantinou, On the complexity of constructing Golomb Rulers. Discrete Appl. Math. 157 (2009) $738-748$.

[16] A. Mucherino, C. Lavor, L. Liberti and N. Maculan, eds., Distance Geometry: Theory, Methods, and Applications. Springer, New York (2013).

[17] O. Oshiga and G. Abreu, Design of orthogonal Golomb rulers with applications in wireless localization. In: Proc. of the 48th Asilomar Conference on Signals, Systems and Computers (2014) 1497-1501.

[18] J. Robinson and A. Bernstein, A class of binary recurrent codes with limited error propagation. IEEE Trans. Inf. Theory 13 (1967) 106-113.

[19] J. Shearer, Some new optimum Golomb rulers. IEEE Trans. Inf. Theory 36 (1990) 183-184.

[20] J. Shearer, Symmetric Golomb squares. IEEE Trans. Inf. Theory 50 (2004) 1846-1847.

[21] S. Sidon, Ein Satz uber trigonometrische polynome und seine anwendungen in der theorie der Fourier-Reihen. Math. Annal. 106 (1932) 536-539.

[22] J. Singer, A theorem in finite projective geometry and some applications to number theory. Trans. Am. Math. Soc. 43 (1938) $377-385$.

[23] M. Slusky and W.-J. van Hoeve, A Lagrangian relaxation for Golomb rulers. Lecture Notes Comput. Sci. 7874 (2013) $251-267$.

\section{Subscribe to Open (S20) A fair and sustainable open access model}

This journal is currently published in open access with no charge for authors under a Subscribe-to-Open model (S2O). Open access is the free, immediate, online availability of research articles combined with the rights to use these articles fully in the digital environment.

$\mathrm{S} 2 \mathrm{O}$ is one of the transformative models that aim to move subscription journals to open access. Every year, as long as the minimum amount of subscriptions necessary to sustain the publication of the journal is attained, the content for the year is published in open access.

\section{Ask your library to support open access by subscribing to this S2O journal.}

Please help to maintain this journal in open access! Encourage your library to subscribe or verify its subscription by contacting subscribers@edpsciences.org

We are thankful to our subscribers and sponsors for making it possible to publish the journal in open access, free of charge for authors. More information and list of sponsors: https://www.edpsciences.org/en/maths-s2o-programme 